\title{
Cuidados de enfermagem no pré-operatório e reabilitação de mastectomia: revisão narrativa da literatura
}

\author{
Nursing care during the preoperative stage and rehabilitation of mastectomy: narrative review of literature
}

Atención de enfermería en el pre-operatorio y rehabilitación de mastectomía: revisión narrativa de la literatura

\section{Pricilla Cândido Alves', Izabel Cristina Falcão Juvenal Barbosa', Joselany Áfio Caetano", Ana Fátima Carvalho Fernandes"}

\author{
' Universidade Federal do Ceará. Faculdade de Farmácia, Odontologia e Enfermagem, \\ Departamento de Enfermagem, Programa de Pós-Graduação em Enfermagem. Fortaleza-CE, Brasil. \\ "Universidade Federal do Ceará. Faculdade de Farmácia, Odontologia e Enfermagem, \\ Departamento de Enfermagem. Fortaleza-CE, Brasil.
}

Submissão: 01/02/2010 Aprovação: 07/11/2010

\section{RESUMO}

Trata-se de uma pesquisa bibliográfica realizada nas bases de dados Lilacs e Scielo, que objetivou revisar a produção científica referente aos cuidados de enfermagem no período pré-operatório e de reabilitação de mastectomia. Foram analisados dezoito artigos focalizados nas seguintes categorias: Cuidados de enfermagem no pré-operatório da mastectomia e O papel da Enfermagem na reabilitação da mulher mastectomizada. Observou-se que os cuidados de enfermagem nos dois períodos são fundamentais para um tratamento mais humanizado. No entanto, estes se encontram escassos e com orientações pouco esclarecedoras, sendo necessário um maior enfoque por parte da equipe com relação ao seu papel e cuidados prestados à mulher nessa condição.

Descritores: Enfermagem; Cuidados de enfermagem; Neoplasias das mamas; Mastectomia.

\section{ABSTRACT}

This is a bibliographic research carried out in Lilacs and Scielo database, which aimed to review the production about nursing care during the preoperative and rehabilitation stages of mastectomy. Eighteen articles were analyzed with focus in these categories: Nursing care in the preoperative mastectomy and The role of nursing in the rehabilitation of women submitted to a mastectomy. It was observed that nursing cares, in the two periods, are a key step for a more human treatment. However, they are scarce and with little enlightening guidance there must be a greater focus on the role by the nursing team in relation to women care.

Descriptors: Nursing; Nursing care; Breast neoplasm; Mastectomy.

\section{RESUMEN}

Este estudio da cuenta de una revisión de la literatura de la atención de enfermería en el pre-operatorio bien como en la rehabilitación de la mastectomía. Fueron analizados dieciocho artículos de acuerdo con las categorías: Cuidados de enfermería en el preoperatorio de mastectomía y El papel de la enfermería en la rehabilitación de las mujeres la mastectomía. Se observó que la atención de enfermería en ambos períodos es crucial para una asistencia más humanizada. Sin embargo, es escasa y con orientaciones poco esclarecedoras, es necesaria una mayor concentración del equipo en relación a su papel en la atención de las pacientes.

Descriptores: Enfermería; Atención de enfermería; Neoplasias de la mama; Mastectomía. 


\section{INTRODUÇÃO}

O câncer de mama pode ser definido como uma doença causada pelo crescimento anormal e desordenado das células que compõem os tecidos da mama, sendo considerado uma patologia temida pela maioria da população feminina, devido à associação com a mutilação física e as alterações que ocorrem no estilo de vida da mulher ${ }^{(1)}$.

Destacamos que, nas últimas décadas, foi registrado um aumento de cerca de dez vezes nas taxas de incidência deste tipo de neoplasia, que aparece como o segundo tipo de câncer mais frequente no mundo e o primeiro entre as mulheres. Segundo a Organização Mundial de Saúde (OMS) estima-se que cerca de 1.050 .000 casos novos de câncer de mama ocorram em todo o mundo. No Brasil, não tem sido diferente, pois, de acordo com informações processadas pelo Instituto Nacional do Câncer (INCA) para 2008, 49.400 casos novos serão registrados; ou seja, há uma taxa bruta de incidência de 50,71 casos para cada 100.000 mulheres, permanecendo estável em relação ao ano anterior ${ }^{(2)}$.

Sabe-se que o câncer de mama está entre as principais causas de morte por câncer nas mulheres, causando um impacto psicológico na percepção da sexualidade, imagem pessoal e autoestima, de uma maneira muito mais significativa que qualquer outro câncer. É importante destacar que a prevenção ainda é a melhor maneira de combater a doença, pois só assim a mesma adquire maiores chances de cura $^{(3)}$.

Outro aspecto importante a ser considerado é o tratamento, visto que, ao mesmo tempo em que trata, o câncer traz inúmeras preocupações para a paciente, nas quais estão inclusas o medo da mutilação e da morte.

Um dos métodos mais utilizados para o tratamento é a mastectomia, uma cirurgia mutiladora que visa remover todo o tumor visível e que pode ter como consequência problemas físicos e psicológicos. A resposta à mutilação é individual e pode estar relacionada a fatores como idade, autoadmiração, estado emocional e situação socioeconômica. Como em qualquer mutilação, a mastectomia requer, além dos cuidados próprios da cirurgia, apoio emocional, objetivando uma melhor compreensão, interação, adaptação e aceitação da autoimagem ${ }^{(4)}$.

A mastectomia é uma intervenção temida e que interfere no estado físico, emocional e social, resultando na mutilação de uma região do corpo que desperta libido e desejo sexual. Esse processo interfere na sexualidade, na autoimagem e na estética feminina hoje em dia muito valorizada e ressaltada. Além dessa dimensão, que simboliza a sexualidade, as mamas ainda são relacionadas a uma importante função, pois, ao produzirem o leite, representam o sustento nos primeiros meses de vida de qualquer ser humano(5).

Portanto, o tratamento do câncer de mama deve ser realizado por uma equipe multidisciplinar, sendo abordado de forma integral e em conjunto, para fornecer melhores subsídios de recuperação à paciente ${ }^{(3)}$.

Desta forma, o enfermeiro exerce importante função neste processo, assumindo o papel de apoio e de promover esforços na busca de uma melhor adaptação da mulher à sua nova situação. Diante disso, cabe ainda ressaltar, que não apenas a equipe de enfermagem, mas toda a equipe multidisciplinar que trabalha com pacientes portadoras de câncer de mama, tem um papel fundamental e cada vez mais necessário não só no acompanhamento pré-operatório, mas também no trans e pós-operatório, bem como na preparação para a alta. Isto deve ocorrer uma vez que, as pacientes afetadas por esta enfermidade precisam ser orientadas juntamente com seus familiares, para que tenham uma compreensão clara dos objetivos do tratamento e suas consequências ${ }^{(6)}$.

Considerando a importância da Enfermagem neste contexto e a falta de esclarecimento e orientações a estas mulheres claramente evidenciadas em diversos estudos é que resolvemos resgatar a produção bibliográfica latino-americana existente a respeito das orientações e cuidados de enfermagem a pacientes que se encontram no período pré-operatório e de reabilitação da mastectomia.

Diante do exposto, objetivou-se, numa perspectiva reflexiva, revisar a produção científica referente aos cuidados de enfermagem no pré-operatório e na reabilitação da mulher submetida à cirurgia de mastectomia, buscando refletir o caráter das orientações e cuidados fornecidos pelo enfermeiro às pacientes que se encontram em tais períodos.

\section{METODOLOGIA}

Para realização do estudo, utilizou-se o método de pesquisa bibliográfica, o qual consiste no exame da bibliografia, para o levantamento e análise do que já foi produzido sobre o assunto que é tema da pesquisa científica ${ }^{(7)}$. Dessa forma, realizou-se o levantamento bibliográfico e, em seguida, a coleta de informações, dados, fatos e informações contidas na bibliografia selecionada.

O levantamento foi realizado em bases de dados da literatura latino-americana, utilizando-se como palavras-chave os termos mastectomia e Enfermagem. Foram encontrados 39 artigos, dos quais 33 pertenciam à base de dados Lilacs (Literatura Latino-Americana e do Caribe em Ciências da Saúde) e seis à Scielo (Scientific Eletronic Library Online). A partir do proposto por Salvador ${ }^{(8)}$, foi feito uma leitura exploratória e seletiva, para verificar se existiam, ou não, informações a respeito do tema proposto e coerentes com os objetivos do estudo.

De acordo com esta leitura, foram selecionados dezoito artigos que abordavam o tema da pesquisa e que foram publicados entre os anos de 1998 a 2008, ou seja, nos últimos dez anos, intervalo selecionado devido ao aumento da incidência e mortalidade por câncer de mama no período ${ }^{(2)}$. Excluiu-se, portanto, 21 artigos, por abordarem temática não pertinente ao objetivo de estudo, tais como uso de prótese mamária, efeitos de tratamentos coadjuvantes, sexualidade, prática de atividades físicas, dentre outras.

A partir dos artigos selecionados, foi realizada uma leitura crítica e interpretativa com a necessária imparcialidade e objetividade, na qual foram relacionadas às informações e ideias dos autores com o objetivo do estudo. A partir da leitura, foram elaboradas categorias reflexivas e descritivas de análise dos dados, configuradas como Cuidados de enfermagem no 
pré-operatório da mastectomia e O papel da Enfermagem na reabilitação da mulher mastectomizada.

\section{RESULTADOS E DISCUSSÃO}

\section{Cuidados de enfermagem no pré-operatório da mastectomia}

O pré-operatório corresponde ao período de tempo que transcorre desde a indicação da cirurgia até a véspera de sua realização, ou seja, $24 \mathrm{~h}$ antes do ato cirúrgico, sendo delimitado, mais precisamente, como o período pré-operatório imediato. Os cuidados pré-operatórios de enfermagem que devem ser prestados durante esse momento, se concentram na intervenção e plano de cuidados para prevenir e minimizar possíveis complicações da cirurgia ${ }^{(9)}$.

Além de cuidados específicos como informações relacionadas com a cirurgia a ser realizada, atividades pré-operatórias gerais e seus fundamentos, comportamentos gerais que são esperados no pós-operatório, imobilização para incisão, exercícios respiratórios, tipos de aparatos a serem usados antes e depois da cirurgia e ambiente esperado para a prática de cuidados ${ }^{(9)}$.

No entanto, o que se observou nos artigos é que pacientes que seriam submetidas à mastectomia enfrentavam um turbiIhão de sentimentos como o medo, a apreensão e preocupação, ressaltados de maneira tão generalizada nos momentos que antecediam a cirurgia e que sinalizam diretamente para a falta de informação e esclarecimento referentes à doença, ao tratamento e à extirpação da mama. e que devem ser trabalhados no pré-operatório, em especial pela equipe de enfermagem, uma vez que esta se encontra diretamente ligada à paciente.

Frente à experiência vivenciada no período pré-operatório, pôde-se constatar o quanto essas mulheres se viam lançadas em um mundo não habitado, o que parecia desencadear-lhes sensações de conflito e aflição, e ocasionar, assim, todo um comportamento de angústia, agitação e medo, o qual poderia ser minimizado se pudessem conhecer e compreender um pouco deste processo.

O desconhecimento do procedimento cirúrgico é, provavelmente, um dos fatores que gera maior insegurança nas muIheres, por implicar mudanças no estilo de vida e ameaça para sua integridade, a que se pode somar a incerteza do inusitado no decorrer da cirurgia e no período que a ela se segue ${ }^{(10)}$.

É válido pontuar que, na iminência da retirada da mama, a desinformação, possui um peso negativo, o que favorece, consideravelmente, a vulnerabilidade, a fragilidade e, até mesmo a falta de apoio a elas no vivenciar deste momento. Neste aspecto, evidencia-se por parte da maioria das mulheres o desconhecimento da anestesia e da cirurgia, os cuidados pré-operatórios e o tempo de permanência na instituição.

Assim, a importância da informação sobre a doença, tratamento e suas consequências são fundamentais. Essas informações e orientações repassadas pelos profissionais da saúde, meios de comunicações e pessoas que vivenciaram a doença, sobre as causas do câncer de mama, aspectos relacionados à prevenção, ao tratamento e suas implicações para a vida da mulher são considerados imprescindíveis para diminuir a ansiedade e o medo frente ao diagnóstico e tratamento ${ }^{(11)}$.
No entanto, percebeu-se que os sujeitos deste processo eram vistos apenas como objetos, e como tal deveriam ser receptores do que lhes era programado. A equipe de saúde direcionava seu fazer apenas para o corpo biológico, de forma dominante, fato que apontou para total nulidade da pessoa como sujeito de seu próprio tratamento.

Dessa forma, percebeu-se nos estudos que a maioria das mulheres possuía uma visão distorcida ou nebulosa frente à realidade que iriam experienciar, visto que Ihes faltava o mínimo de esclarecimento e orientação sobre o momento vivido.

O "medo do desconhecido" foi apontado nos estudos como a maior fonte de ansiedade em pacientes com câncer de mama a serem submetidas a procedimento cirúrgico. Existem inúmeros fatores identificados como responsáveis por esta ansiedade que cerca o momento do ato anestésico-cirúrgico como: preocupações com lesões que possam ocorrer durante o procedimento cirúrgico, receio de dor no período pós-operatório, separação da família, perda da independência, medo de ficar incapacitada, medo de acordar no meio da anestesia, medo do diagnóstico e das complicações ${ }^{(12)}$.

Além desses sentimentos, a ausência de orientações sobre o real estado de saúde, os procedimentos terapêuticos e as finalidades do tratamento também estiveram presentes e acabavam agravando as preocupações e dificultando a aceitação das mudanças ocorridas ${ }^{(6)}$.

Com isso, infere-se que a questão da participação da muIher com câncer de mama no seu processo de tratamento, inclusive nas modalidades a que será submetida, deve ser conduzida dentro de uma proposta de um modelo assistencial traçado pela ética do cuidar de cidadãos, de forma a permitir que esta se torne sujeito de participação de sua própria assistência.

Diferentemente do que se observou nos artigos, onde os cuidados de enfermagem no período que antecedia a mastectomia encontravam-se escassos, com orientações pouco esclarecedoras e bastante desvirtuadas daquilo que seria considerado como ideal ou pelo menos suficiente, uma vez que se identificou que as mulheres quase não recebiam informações ou orientações significativas por parte da equipe de enfermagem, e quando esta existia referia-se apenas à extirpação da mama.

Para o tratamento cirúrgico se tornar mais humano a paciente necessita ser mais bem preparada emocionalmente e as orientações devem ser completas. Não se trata apenas de falar o nome da cirurgia e sim de explicar cada passo, ou seja, do que, por que, quando, por quem, como, onde e as consequências, a fim de que a paciente possa estar ciente do tratamento e colaborar na recuperação ${ }^{(10)}$.

A assistência de enfermagem deve incluir medidas para prevenir ou minimizar a angústia referida pela mulher após o diagnóstico de câncer de mama e posterior tratamento, e que incluem a mobilização de suporte social disponível, a ênfase nas questões psicossociais e o fornecimento de informações à mulher, no sentido de facilitar o enfrentamento efetivo da doença e procedimento cirúrgico, restaurando o senso de normalidade e independência física ${ }^{(13)}$.

A visita pré-operatória, portanto, deve caracterizar-se por uma atuação expressiva do enfermeiro, que é entendida por 
apoio emocional, presença e atenção, aspectos tão característicos da arte do cuidar em enfermagem introduzida por Florence Nightingale. Tal atitude visa evitar o cuidar totalmente mecanizado e fragmentado, bem como o surgimento tanto de complicações físicas quanto psíquicas ${ }^{(14)}$.

Além disso, estes profissionais devem na visita pré-operatória reconhecer as necessidades, os anseios e os desejos sentidos pelas mulheres, de forma que ao analisá-los, possa-se traçar um plano de cuidados individualizado e eficaz, onde os enfermeiros apoiados nesse método possam adotar estratégias de assistência sistematizada, com economia de tempo e grande eficácia na recuperação destas mulheres.

\section{O papel da Enfermagem na reabilitação da mulher mastectomizada}

No Brasil, o diagnóstico de câncer de mama, ainda é feito, na maioria das vezes, quando a doença se encontra em estágios avançados e a conduta terapêutica mais empregada tem sido a do tratamento cirúrgico radical, representada pela mastectomia, que demanda uma série de cuidados voltados para recuperação física, emocional e social das mulheres que enfrentam este processo ${ }^{(15)}$.

O período pós-operatório é marcado pela ambivalência, ou seja, há o alívio de ter sobrevivido à cirurgia e a esperança de estar curada. Mas também há o medo do retorno da doença, de enfrentar a dor e os curativos, de enfrentar a possibilidade permanente de um corpo mutilado e, ainda, preocupações com a feminilidade e com as reações do companheiro frente à mastectomia ${ }^{(10)}$.

As dificuldades enfrentadas pela mulher após a mastectomia são muitas, especialmente, pelo comprometimento da autoimagem tanto da própria cirurgia e dos efeitos colaterais da quimioterapia adjuvante, como do preconceito e da dor e das dificuldades físicas que acarreta. Com isso, é evidente a importância do trabalho da Enfermagem na reabilitação da mulher mastectomizada, para amenizar essas dificuldades e fortalecê-la para o seu enfrentamento de maneira menos traumática ${ }^{(16)}$.

A primeira grande dificuldade a ser enfrentada pelas muIheres, após a mastectomia, é sua própria aceitação, como de olhar-se no espelho e aceitar que seu corpo está diferente, sem uma parte que culturalmente representa a feminilidade. A identificação da mutilação se dá pela percepção da assimetria do corpo e pela visibilidade da cirurgia, o que para muitas, é um momento agressivo a sua autoimagem ${ }^{(16)}$.

Tal fato reflete-se nas limitações que a mulher irá enfrentar em situações que envolvem a exposição do corpo e ajuste social para realização de suas atividades. Por isso, é de grande importância o papel da Enfermagem na tentativa de resgatar o conceito que a mulher mastectomizada tem de si mesma ${ }^{(17)}$.

O preconceito social é motivo de constrangimento para estas mulheres, dificultando, ainda mais, o enfrentamento desta vivência. Isto contribui para que estas pacientes sejam preconceituosas em relação ao seu próprio corpo, o que leva a outra dificuldade a ser enfrentada no pós-operatório: o retorno a vida sexual. A maioria delas tem vergonha de mostrar-se nua na frente de seus parceiros, preferindo, então, manter relações sexuais com um sutiã ou camiseta ${ }^{(16)}$.
Neste momento, além da atuação profissional é fundamental para recuperação destas mulheres o apoio recebido pela família e rede social, uma vez que as mudanças ocorridas após o tratamento da doença são significativas e transformadoras. Há necessidade de apoio por parte de pessoas próximas, como esposo, filhos, amigos, enfim, alguém que possa ajudá-las nesse processo ${ }^{(18)}$.

Assim, a reabilitação da mulher submetida à cirurgia de câncer de mama requer um suporte integral, que envolva tanto a família, como uma assistência multiprofissional, na qual é de grande importância o papel da Enfermagem. Nesse processo de reabilitação a mulher deverá receber informações a respeito dos cuidados após a cirurgia, orientações sobre as diferentes etapas de recuperação, cuidados com o membro superior homolateral à cirurgia, exercícios que recuperem a capacidade funcional do braço e do ombro, além de informações sobre outros tratamentos como quimioterapia, radioterapia e hormonioterapia ${ }^{(19)}$.

Para alcançar esta reabilitação, torna-se necessário o desenvolvimento pela equipe de enfermagem de ações educativas que ajudem as pacientes e seus cuidadores a adquirirem conhecimento e habilidades sobre os diversos aspectos do seu autocuidado ${ }^{(13)}$. Entretanto, pouco se tem produzido sobre as estratégias de cuidado adotadas pela Enfermagem à mulher mastectomizada, as quais, sem dúvida alguma, contribuem para o sucesso do tratamento.

A principal atividade que se observou nos estudos foram as atividades grupais, com as quais se busca promover a autoestima da mulher, contribuindo para sua qualidade de vida. Os grupos de apoio são uma estratégia inovadora que vem sendo construída e aprimorada a cada dia, sendo constituídos por uma equipe multiprofissional (enfermeiro, psicólogo, terapeuta ocupacional, etc), que planeja atividades visando à promoção e recuperação da saúde ${ }^{(16)}$.

A Enfermagem exerce fundamental importância nos trabalhos realizados nestes grupos, pois é papel do enfermeiro ensinar o autocuidado; valorizar o indivíduo como ser único, com seus medos e suas dúvidas visando promover um crescimento individual a partir da aceitação do indivíduo como ser único e singular, dando-lhe estímulo e apoio.

Satisfatoriamente, há o desejo de participação destas muIheres no autocuidado após a cirurgia, porém, infelizmente houve pouca informação e suporte profissional para que isso ocorresse, o que leva a crer que a equipe de saúde apoia insuficientemente a recuperação da paciente. $\mathrm{O}$ que as mulheres esperam após a hospitalização é receber encorajamento, reabilitação mental, ajuda para desenvolver uma atitude positiva em relação à doença e seu tratamento, e apoio dos profissionais de saúde, parentes e amigos ${ }^{(10)}$.

Além disso, observou-se que as pacientes desejavam compartilhar com as enfermeiras suas dúvidas, suas tristezas, sua desesperança e também suas angústias. E, nesse compartiIhar, esperavam receber o suporte necessário para enfrentar sua nova condição. No entanto, no que concerne ao suporte informativo, as mulheres expressavam sentimentos de insegurança e revolta por não terem recebido orientações suficientes nos serviços para continuidade do tratamento. 
Tais dados chamam a atenção para que a equipe de enfermagem esteja mais atenta quanto ao seu papel no preparo e nos cuidados prestados a paciente durante o processo de reabilitação, o qual deve ser iniciado e trabalhado desde a fase de diagnóstico, visando o mínimo de complicações e perdas possíveis para estas clientes.

Este papel envolve uma importante missão, pois o enfermeiro, enfrenta o desafio no cuidado prestado de minimizar o sofrimento da mulher, ou seja, seu trabalho requer valorizá-la e estimulá-la a transformar seu medo em força de sobrevivência, favorecendo sua adesão e participação na reabilitação, no autocuidado durante o decorrer do tratamento ${ }^{(16)}$.

Assim, a Enfermagem necessita traçar um plano de cuidados à mulher que ofereça suporte informativo em relação ao câncer, tratamento e reabilitação, que ofereça mais conforto e tranquilidade, estimule a expressão de sentimentos, a participação em grupos de autoajuda, de maneira a minimizar o drama experienciado pela mulher que vivencia a mastectomia.

\section{CONSIDERAÇÕES FINAIS}

A pesquisa bibliográfica possibilitou a percepção de que a mastectomia provoca reações de incertezas gerando angústia frente ao desconhecido, a falta de confiança e a expectativa do "vir a ser". Portanto, o enfermeiro deve ajudar a cliente a superar tais sensações, atentando para a linguagem verbal e não verbal da mulher, orientando-a sobre seus anseios e preocupando-se com a melhoria da qualidade de vida. Desta forma, há a possibilidade de exercer plenamente o ato de cuidar e possibilitar a superação dos obstáculos.

Nessa perspectiva, nota-se que a mulher passa por um pré-operatório estressante, incerto e que gera medo. Assim, ressaltamos a importância do papel educativo e de apoio emocional durante a assistência de enfermagem, bem como a inserção da cliente no processo decisório de seu tratamento, uma vez que ela tem o direito de decidir, opinar e saber como vai cuidar de seu corpo.

As orientações recebidas pelas mulheres no período pré-operatório e de reabilitação são precárias, o que vai de encontro ao que se espera, pois o profissional de saúde que se propõe a trabalhar com mulheres com câncer de mama, deve prestar assistência que congregue técnica, ciência e humanização, fornecendo todas as informações e orientações, respeitando as necessidades e o nível de entendimento dessas mulheres, reabilitando-as para o autocuidado.

Assim, a mulher submetida a uma mastectomia deve ser bem orientada no seu pré-operatório e reabilitada após a cirurgia em seus vários aspectos, tanto físico, emocional, social e profissional. A superação de certas dificuldades pode ser minimizada pelos os enfermeiros e demais equipe, que percebam e compreendam o seu drama e sejam conhecedores desta causa, já que o câncer de mama é um agravo de incidência significativa na saúde de nosso país e, também a nível mundial.

\section{REFERÊNCIAS}

1. Kucharsk FA. Perfil de mulheres na detecção precoce do câncer de mama. Fortaleza (CE): Universidade Federal do Ceará; 2003.

2. Ministério da Saúde (BR). Instituto Nacional do Câncer (INCA). Estimativas de incidência e mortalidade por câncer no Brasil. [online] [citado 2008 jul 25]. Disponível em: http:// www.hospcancer-ICC.org.br/conteudo.php.

3. Carvalho CMRG, Brito CMS, Nery IS, Figueiredo MLS. Prevenção de câncer de mama em mulheres idosas: uma revisão. Rev Bras Enferm. 2009;62(4):579-82.

4. Perlini NMOG, Bervian PI. A família (con)vivendo com a mulher/mãe após a mastectomia. Rev Bras Cancerol 2006;52(2):121-28.

5. Pinho LS, Campos ACS, Fernandes AFC, Lobo AS. Câncer de mama: da descoberta à recorrência da doença. Rev Eletrôn Enf [serial online] 2007 [citado 2008 jul 25];9(1):154165. Disponível em: www. fen.ufg.br/revista/v9/n1/ v9n1a12.htm.

6. Bittencourt JFV, Cadete MMM. Vivências da mulher a ser mastectomizada: esclarecimentos e orientações. Rev Bras Enferm 2002;55(4):420-23.

7. Ruiz JA. Metodologia científica: guia para eficiência nos estudos. São Paulo (SP): Atlas; 1992.

8. Salvador AD. Métodos e técnicas de pesquisa bibliográfica. Porto Alegre (RS): Sulina; 1986.
9. Barbosa ICFJ, Fernandes AFC, Silva RM. Gurgel AL. Benefício de la terapia grupal em la rehabilitácion de mujeres mastectomizadas. Rev Enferm IMSS 2002 [serial online] [citado 2008 mai 20]. 10(2):79-84 Available from: http:// www.imss.gob.mx/NR/rdonlyres/F79B0887-CFB4-4421-927B-6F3B3E77D07E/0/BenefReha10.pdf

10. Camargo TC, Souza IEO. Atenção à mulher mastectomizada: discutindo os aspectos ônticos e a dimensão ontológica da atuação da enfermeira do Hospital do Câncer III. Rev. Latino-Am. Enfermagem 2003; 11(5):614-21.

11. Duarte TP, Andrade AC. Enfrentando a mastectomia: análise dos relatos de mulheres mastectomizadas sobre questões ligadas à sexualidade. Estud Psicol 2003;8(1):155-63.

12. Alves MLM, Pimentel AJ, Guaratini AA, Marcelino JAM, Gozanni JL, Mathias LAST. Ansiedade no período pré-operatório de cirurgias de mama: estudo comparativo entre pacientes com suspeita de câncer e a serem submetidas a procedimentos estéticos. Rev Bras Anestesiol 2007;57(2):147-56.

13. Gualda DMR, Bergamasco RB. Enfermagem, cultura e o processo saúde-doença. São Paulo (SP): Ícone; 2004.

14. Garcia HF. Compreendendo a necessidade do paciente com câncer de receber orientações para a cirurgia: Implicações da visita pré-operatória pelo enfermeiro. Rev Bras Cancerol 1999;45(2):15-26. 
15. Guttiérrez MGR, Pinelli FGS, Areias VL, Barbi T. Avaliação de um programa educativo sobre os cuidados com o sistema de drenagem para cuidadores de muIheres mastectomizadas. Acta paul. enferm. 2004;17 (4):412-18.

16. Pereira SG, Rosenhein DP, Bulhosa MS, Lunard VL, FiIho WDL. Vivências de cuidados da mulher mastectomizada: uma pesquisa bibliográfica. Rev Bras Enferm 2006;59(6):791-5.
17. Talhaferro B, Lemos SS, Oliveira E. Mastectomia e suas conseqüências na vida da mulher. Arq Ciênc Saúde 2007; 14(1): 412-18.

18. Barbosa RCM, Ximenes LB, Pinheiro AKB. Mulher mastectomizada: desempenho de papéis e redes sociais de apoio. Acta Paul Enferm 2004;17(1):18-24.

19. Mamede MV, Clapis MJ, Panobianco MS, Biffi RG, Bueno LV. Orientações pós-mastectomia: o papel da enfermagem. Rev Bras Cancerol 2000;46(1):57-62. 